\title{
Exponential Type Estimators Using Sub-Sampling Method with Applications in Agriculture
}

\author{
Ceren ÜNAL $^{\mathrm{a}^{*}}$ (D) Cem KADILAR ${ }^{\mathrm{b}}$ (D) \\ ${ }^{a}$ Hacettepe University, Institute of Science, Beytepe, Ankara, TURKEY \\ ${ }^{b}$ Department of Statistics, Hacettepe University, 06800 Beytepe, Ankara, TURKEY

\section{ARTICLE INFO} \\ Research Article \\ Corresponding Author: Ceren ÜNAL, E-mail: cerenunal@hacettepe.edu.tr \\ Received: 14 April 2020 / Revised: 29 August 2021 / Accepted: 01 September 2021 / Online: 01 September 2022 \\ Cite this article \\ ÜNAL C, KADILAR C (2022). Exponential Type Estimators Using SUB-Sampling Method with Applications in Agriculture. Journal of Agricultural Sciences (Tarim Bilimleri Dergisi), \\ 28(3):457-472. DOI: 10.15832/ankutbd.915999
}

\section{ABSTRACT}

In this article, the family of exponential type estimators with the auxiliary variable is proposed in the case of non-response scheme for the purpose of obtaining the unknown population mean of the study variable. The nonresponse scheme is examined under two main cases as Case I and Case II. The bias, mean square error (MSE) and minimum MSE of the proposed family of estimators are obtained in detail for both cases. After theoretical inferences, empirical studies are carried out to show the appropriateness of the proposed family of estimators in the field of agriculture. The MSE and PRE (Percentage Relative Efficiency) values are obtained. According to the results, the proposed estimators provide more efficient results than existing estimators in the literature under the obtained conditions for both cases. We conclude that the proposed family of estimators can be applied to the agriculture data successfully.

Keywords: Agriculture, Efficiency, Non-response, Population mean estimator, Simple random sampling

\section{Introduction}

Instead of population, working on a sample plays an important role in terms of time, money, and labor force. The parameters of the population, such as total, mean, proportions and variance can be obtained via estimators in sample surveys. Here, the main aim is to propose more efficient estimator than other estimators in literature. For this reason, the information of the auxiliary variable is used extensively in order to obtain the efficient results. In literature, we may see various types of estimators, such as ratio, regression, product, exponential and so on in the presence of the auxiliary variable. In these types of estimators, if the relation between study $(y)$ and auxiliary variable $(x)$ is a straight line passing through the origin, the usual regression, ratio and product types of estimators have equal efficiencies. However, when the line does not pass through the origin, the regression, ratio and product types of estimators do not have equal efficiencies (Solanki et al. 2012). For this reason, exponential type of estimators becomes prominent among others.

In general, the estimators are proposed when all information on variables is present. However, some units on several variables cannot be obtained all the time. This situation is the most important problem in the sample survey that is named as non-response (Dansawad 2019). In order to deal with this situation, Hansen and Hurwitz (1946) introduced a new technique of sub-sampling. Here, the main aim is to reduce the effect of non-response using both response and non-response units in the estimator.

In this technique, from a population size of $N$ units, as $S=\left(S_{1}, S_{2}, \ldots, S_{N}\right)$, a sample of size $n$ units is drawn. However, the population size $N\left(N_{1}+N_{2}=N\right)$ is divided into two groups, $\mathrm{N}_{1}$ and $\mathrm{N}_{2}$, as respondent and non-respondent units, respectively. Similarly, the response units are available only on $n_{l}$ while $n_{2}\left(n_{2}=n-n_{1}\right)$ units are obtained as non-response. Due to the extra effort, a sub-sample size of $r=\frac{n_{2}}{z}(z>1)$ is drawn from $\mathrm{n}_{2}$. Thus, the population mean can be estimated by using $\left(n_{1}+r\right)$ units, instead of $n$ units, in this sub-sampling technique. Note that $z$ is the inverse of the sampling rate whose different values are used for the MSE and PRE values.

The unbiased estimator is defined by Hansen and Hurwitz (1946) to estimate the population mean for the first time in the sub-sampling technique as follows: 
$t_{H}=w_{1} \bar{y}_{1}+w_{2} \bar{y}_{2(r)}$,

whose variance is given by

$V\left(t_{H}\right)=\bar{Y}^{2}\left(\lambda C_{y}^{2}+\frac{W_{2}(z-1)}{n} C_{y(2)}^{2}\right)$

In Equation (1), $w_{1}=\frac{n_{1}}{n}$ and $w_{2}=\frac{n_{2}}{n}$ refer the weights of response and non-response units for the sample, respectively. Besides, $\bar{y}_{1}$ and $\bar{y}_{2(r)}$ denote the sample means of $y$ based on $n_{1}$ and $r\left(r=n_{2} / z\right)$ units. In Equation (2), $f=\frac{n}{N}, \lambda=\frac{1-f}{n}, C_{y}^{2}=\frac{S_{y}^{2}}{\bar{Y}^{2}}$ and $C_{y(2)}^{2}=S_{y(2)}^{2} / \bar{Y}^{2}$. In addition, $W_{2}=N_{2} / N$ is the weight of $N_{2}$ units in the population.

The non-response situation is examined under Case I and Case II, separately. In Case I, units of non-response exist only on $y$. In Case II, units of non-response exist on both $y$ and $x$. In addition to this, the population mean of $x$ is known for both cases.

After the sub-sampling method and pioneer study of Hansen and Hurwitz (1946), many estimators have been proposed in the literature considering the non-response scheme. Some of the estimators for the population mean are given in Tables 1 and 2 , according to the Cases I and II, respectively.

Using the sub-sampling method, Rao (1986) proposed the classical ratio $\left(t_{R 1}\right)$ and regression $\left(t_{\text {reg1 }}\right)$ estimators under the Case I. Singh et al. (2009) first defined the exponential type estimator $\left(t_{\text {exp } 1}\right)$ by adapting the estimator proposed by Bahl and Tuteja (1991) to the Case I. Following these estimators, Olufadi and Kumar (2014), Yadav et al. (2016), Kumar and Kumar (2017), Pal and Singh (2016, 2017, 2018), Dansawad (2019), Singh and Usman (2019a, 2019b), Unal and Kadilar (2021) proposed various estimators taking the advantage of the exponential function.

In these estimators, $\bar{y}^{*}$ is the sample mean of $y$ under the non-response scheme. Also, $\bar{x}$ and $\bar{X}$ are the sample and the population means of $x$, respectively, while $\bar{Y}$ is the population mean of $y$.

Under the Case II, Cochran (1977) defined the ratio $\left(t_{R 2}\right)$ and regression $\left(t_{\text {reg } 2}\right)$ estimators while Singh et al. (2009) proposed the exponential type estimator for the population mean. Following these estimators, Kumar and Bhougal (2011), Kumar (2013), Yadav et al (2016), Kumar and Kumar (2017), Pal and Singh (2016, 2017, 2018), Singh and Usman (2019a, 2019b), Unal and Kadilar (2020, 2021) and Riaz et al. (2020) proposed estimators using the exponential function for the population mean for the Case II.

Note that in Tables 1 and 2, $\bar{x}^{*}$ refers the sample mean of $x$ in the case of non-response and the coefficient of the population correlation between $x$ and $y$ for the non-response group is referred as $\rho_{y x(2)}$. 
Table 1- Existing estimators in literature for the Case I

\begin{tabular}{|c|c|}
\hline Authors & Estimators \\
\hline Rao (1986) & $t_{R 1}=\bar{y}^{*} \frac{\bar{X}}{\bar{x}}$ \\
\hline Rao (1986) & $t_{\text {reg } 1}=\bar{y}^{*}+b^{*}(\bar{X}-\bar{x}), b^{*}=S_{x y}^{*} / S_{x}^{* 2}$ \\
\hline Singh et al. (2009) & $t_{\mathrm{exp} 1}=\bar{y}^{*} \exp \left(\frac{\bar{X}-\bar{x}}{\bar{X}+\bar{x}}\right)$ \\
\hline Olufadi and Kumar (2014) & $t_{Y K 1}=\bar{y}^{*}\left\{\alpha \exp \left(\frac{\bar{X}-\bar{x}}{\bar{X}+\bar{x}}\right)+(1-\alpha) \exp \left(\frac{\bar{x}-\bar{X}}{\bar{x}+\bar{X}}\right)\right\}$ \\
\hline Yadav et al. (2016) & $t_{Y 1}=\bar{y}^{*} \exp \left(\frac{\bar{X}-\bar{x}}{\bar{X}+(\alpha-1) \bar{x}}\right)$ \\
\hline Pal and Singh (2016) & $t_{(\alpha, \delta), 1}=\bar{y}^{*}\left(\frac{\bar{X}}{\bar{x}}\right)^{\alpha} \exp \left\{\left(\frac{\delta(\bar{X}-\bar{x})}{\bar{x}+\bar{X}}\right)\right\}$ \\
\hline Pal and Singh (2017) & $t_{P S 1}=\alpha \bar{y}^{*}\left(\frac{\bar{X}}{\bar{x}}\right)+(1-\alpha) \bar{y}^{*} \exp \left(\frac{\bar{X}-\bar{x}}{\bar{X}+\bar{x}}\right)$ \\
\hline Kumar and Kumar (2017) & $t_{K K 1}=d_{1}^{*} \bar{y}^{*} \exp \left(\frac{\bar{X}-\bar{x}}{\bar{X}+\bar{x}}\right)$ \\
\hline Pal and Singh (2018) & $t_{S P 1}=\bar{y}^{*}\left[\eta\left\{\frac{(1-\delta) \bar{x}+\delta \bar{X}}{\delta \bar{x}+(1-\delta) \bar{X}}\right\}+(1-\eta)\left\{\frac{\delta \bar{x}+(1-\delta) \bar{X}}{(1-\delta) \bar{x}+\delta \bar{X}}\right.\right.$ \\
\hline Dansawad (2019) & $t_{D}=\bar{y}^{*} \exp \left(\frac{(a \bar{X}+b)-(a \bar{x}+b)}{(a \bar{X}+b)+(a \bar{x}+b)}\right)$ \\
\hline Singh and Usman (2019a) & $t_{U S 1}=\bar{y}^{*}\left(\frac{\bar{X}}{\bar{x}}\right)^{\alpha} \exp \left\{\left(c \frac{\bar{X}-\bar{x}}{\bar{X}+\bar{x}}\right)\right\}$ \\
\hline Singh and Usman (2019b) & $t_{S U 1}=\left[d_{1}^{*} \bar{y}^{*}+d_{2}^{*}(\bar{X}-\bar{x})\right] \exp \left(\frac{\bar{X}-\bar{x}}{\bar{X}+\bar{x}}\right)$ \\
\hline Unal and Kadilar (2021) & $t_{1, i}=k \bar{y}^{*}\left(\frac{a \bar{X}+b}{a \bar{x}+b}\right)^{c} \exp \left(\frac{a(\bar{X}-\bar{x})}{a(\bar{X}+\bar{x})+2 b}\right.$ \\
\hline
\end{tabular}


Table 2- Existing estimators in literature for the Case II

\begin{tabular}{|c|c|}
\hline Authors & Estimators \\
\hline Cochran (1977) & $t_{R 2}=\bar{y}^{*} \frac{\bar{X}}{\bar{x}^{*}}$ \\
\hline Cochran (1977) & $t_{\text {reg } 2}=\bar{y}^{*}+b^{*}\left(\bar{X}-\bar{x}^{*}\right)$ \\
\hline Singh et al. (2009) & $t_{\mathrm{exp} 2}=\bar{y}^{*} \exp \left(\frac{\bar{X}-\bar{x}^{*}}{\bar{X}+\bar{x}^{*}}\right)$ \\
\hline Kumar and Bhougal (2011) & $t_{K B}=\bar{y}^{*}\left\{\alpha \exp \left(\frac{\bar{X}-\bar{x}^{*}}{\bar{X}+\bar{x}^{*}}\right)+(1-\alpha) \exp \left(\frac{\bar{x}^{*}-\bar{X}}{\bar{x}^{*}+\bar{X}}\right)\right\}$ \\
\hline Kumar (2013) & $t_{K}=\bar{y}^{*} \exp \left(\frac{(a \bar{X}+b)-\left(a \bar{x}^{*}+b\right)}{(a \bar{X}+b)+\left(a \bar{x}^{*}+b\right)}\right)$ \\
\hline Yadav et al. (2016) & $t_{Y 2}=\bar{y}^{*} \exp \left(\frac{\bar{X}-\bar{x}^{*}}{\bar{X}+(\alpha-1) \bar{x}^{*}}\right)$ \\
\hline Pal and Singh (2016) & $t_{(\alpha, \delta), 2}=\bar{y}^{*}\left(\frac{\bar{X}}{\bar{x}^{*}}\right)^{\alpha} \exp \left\{\left(\frac{\delta\left(\bar{X}-\bar{x}^{*}\right)}{\bar{X}+\bar{x}^{*}}\right)\right\}$ \\
\hline Pal and Singh (2017) & $t_{P S 2}=\alpha \bar{y}^{*}\left(\frac{\bar{X}}{\bar{x}^{*}}\right)+(1-\alpha) \bar{y}^{*} \exp \left(\frac{\bar{X}-\bar{x}^{*}}{\bar{X}+\bar{x}^{*}}\right)$ \\
\hline Kumar and Kumar (2017) & $t_{K K 2}=d_{1}^{*} \bar{y}^{*} \exp \left(\frac{\bar{X}-\bar{x}^{*}}{\bar{X}+\bar{x}^{*}}\right)$ \\
\hline Pal and Singh (2018) & $t_{S P 2}=\bar{y}^{*}\left[\eta\left\{\frac{(1-\delta) \bar{x}^{*}+\delta \bar{X}}{\delta \bar{x}^{*}+(1-\delta) \bar{X}}\right\}+(1-\eta)\left\{\frac{\delta \bar{x}^{*}+(1-\delta) \bar{X}}{(1-\delta) \bar{x}^{*}+\delta \bar{X}}\right.\right.$ \\
\hline Singh and Usman (2019a) & $t_{U S 2}=\bar{y}^{*}\left(\frac{\bar{X}}{\bar{x}^{*}}\right)^{\alpha} \exp \left\{c \frac{\bar{X}-\bar{x}^{*}}{\bar{X}+\bar{x}^{*}}\right\}$ \\
\hline Singh and Usman (2019b) & $t_{S U 2}=\left[d_{1}^{*} \bar{y}^{*}+d_{2}^{*}\left(\bar{X}-\bar{x}^{*}\right)\right] \exp \left(\frac{X-\bar{x}^{*}}{\bar{X}+\bar{x}^{*}}\right)$ \\
\hline Unal and Kadilar (2020) & $t_{U K}=\bar{y}^{*}\left(\frac{\bar{x}}{\bar{X}}\right) \exp \left(\frac{X-\bar{x}}{\bar{X}+\bar{x}^{*}}\right)$ \\
\hline Riaz et al. (2020) & $t_{R N A Q}=\bar{y}^{*}\left[d_{1}^{*}+d_{2}^{*}\left(a \bar{X}-a \bar{x}^{*}\right)\right] \exp \left(\frac{c\left(a \bar{X}-a \bar{x}^{*}\right)}{\left(a \bar{X}-a \bar{x}^{*}\right)+2 b}\right)$ \\
\hline Unal and Kadilar (2021) & $t_{2, i}=k \bar{y}^{*}\left(\frac{a \bar{X}+b}{a \bar{x}^{*}+b}\right)^{c} \exp \left(\frac{a\left(\bar{X}-\bar{x}^{*}\right)}{a\left(\bar{X}+\bar{x}^{*}\right)+2 b}\right.$ \\
\hline
\end{tabular}

In Tables $1-2, \alpha, d_{1}^{*}, d_{2}^{*}, k, \eta$ and $\delta$ are chosen constants that make MSE minimum and $c$ takes the $(0,-1,1)$ values. Besides, $a$ and $b$ are either function of the known parameters of $x$ or real numbers.

For this study, our main motivation is proposing more efficient estimator than existing estimators in literature in the presence of both non-response schemes as Case I and Case II. In this article, we introduce the proposed family of estimators for both cases. The appropriateness of the proposed family of estimators is examined theoretically and numerically on agriculture in Sections 4 and 5, respectively. Finally, the article is concluded with the obtained results. 


\section{Proposed Family of Estimators}

Singh et al. (2020) proposed a new estimator using the exponential function. Based on this estimator, a new family of estimators for the population mean is proposed under the non-response scheme, Case I and Case II, respectively, as follows:

\subsection{CASE I:}

The first family of estimators is defined as

$t_{C 1, j}=\bar{y}^{*}\left(v_{1}+v_{2} \frac{\bar{X}}{\bar{x}}\right) \exp \left(\frac{\phi(\bar{X}-\bar{x})}{\phi(\bar{X}+\bar{x})+2 \varphi}\right), j=1,2, \ldots, 10$,

Where; $v_{1}$ and $v_{2}$ are constants that make the $\operatorname{MSE}\left(t_{C 1, j}\right), j=1,2, \ldots, 10$ minimum. Besides, $\phi$ and $\varphi$ are either functions of the known parameters of $x$ or real numbers.

In order to obtain the bias and MSE of the $t_{C 1, j}, j=1,2, \ldots, 10$, we use the following notations as

$\bar{y}^{*}=\left(\bar{Y} e_{y}^{*}+\bar{Y}\right), \bar{x}=\left(\bar{X} e_{x}+\bar{X}\right), \quad E\left(e_{x}\right)=0, E\left(e_{x}^{2}\right)=\lambda C_{x}^{2}, E\left(e_{y}^{*}\right)=0, E\left(e_{y}^{* 2}\right)=\left(\lambda C_{y}^{2}+\frac{W_{2}(z-1)}{n} C_{y(2)}^{2}\right)$ $E\left(e_{y}^{*} e_{x}\right)=\lambda C_{y x}$,

Where; $C_{x}^{2}=\frac{S_{x}^{2}}{\bar{X}^{2}}, C_{x y}=\rho_{x y} C_{x} C_{y}, C_{x(2)}^{2}=\frac{S_{x(2)}^{2}}{\bar{X}^{2}}, C_{y x(2)}=\rho_{y x(2)} C_{x(2)} C_{y(2)}$ and $\rho_{x y}$ is the coefficient of the population correlation between $y$ and $x$.

We re-write the Equation (3) using these notations as follows:

$$
\begin{aligned}
t_{C 1, j} & =\bar{Y}\left(1+e_{y}^{*}\right)\left(v_{1}+v_{2} \frac{\bar{X}}{\bar{X}\left(1+e_{x}\right)}\right)\left(1-\vartheta e_{x}+\frac{3 \vartheta^{2}}{2} e_{x}^{2}\right) \\
& =\bar{Y}\left(1+e_{y}^{*}\right)\left(v_{1}-v_{1} \vartheta e_{x}+\frac{3 v_{1} \vartheta^{2}}{2} e_{x}^{2}+v_{2}-v_{2} \vartheta e_{x}+\frac{3 v_{2} \vartheta^{2}}{2} e_{x}^{2}-v_{2} e_{x}+v_{2} \vartheta e_{x}^{2}+v_{2} e_{x}^{2}\right),
\end{aligned}
$$

Where; $\vartheta=\frac{\phi \bar{X}}{2(\phi \bar{X}+\varphi)}$.

Using different $\phi$ and $\varphi$ values, we can propose various estimators and some members of the proposed estimators are given in Table 3. 
Table 3- Some members of the $t_{C 1, j}, j=1,2, \ldots, 10$

\begin{tabular}{|c|c|c|c|c|}
\hline \multicolumn{3}{|c|}{ Values } & \multirow{2}{*}{\multicolumn{2}{|c|}{ Estimators }} \\
\hline$\vartheta_{i}$ & $\phi$ & $\varphi$ & & \\
\hline$\vartheta_{1}=\frac{\bar{X}}{2(\bar{X}+1)}$ & 1 & 1 & $t_{C 1,1}=\bar{y}^{*}\left(v_{1}+v_{2} \frac{\bar{X}}{\bar{x}}\right) \exp$ & $\left.\frac{(\bar{X}-\bar{x})}{(\bar{X}+\bar{x})+2}\right)$ \\
\hline$\vartheta_{2}=\frac{\bar{X}}{2\left(\bar{X}+\beta_{2}(x)\right)}$ & 1 & $\beta_{2}(x)$ & $t_{C 1,2}=\bar{y}^{*}\left(v_{1}+v_{2} \frac{\bar{X}}{\bar{x}}\right) \exp$ & $\left.\frac{(\bar{X}-\bar{x})}{(\bar{X}+\bar{x})+2 \beta_{2}(x)}\right)$ \\
\hline$\vartheta_{3}=\frac{\bar{X}}{2\left(\bar{X}+C_{x}\right)}$ & 1 & $C_{x}$ & $t_{C 1,3}=\bar{y}^{*}\left(v_{1}+v_{2} \frac{\bar{X}}{\bar{x}}\right) \exp$ & $\left.\frac{(\bar{X}-\bar{x})}{(\bar{X}+\bar{x})+2 \varphi}\right)$ \\
\hline$\vartheta_{4}=\frac{\bar{X}}{2(\bar{X}+\rho)}$ & 1 & $\rho$ & $t_{C 1,4}=\bar{y}^{*}\left(v_{1}+v_{2} \frac{\bar{X}}{\bar{x}}\right) \exp$ & $\left.\frac{(\bar{X}-\bar{x})}{(\bar{X}+\bar{x})+2 \rho}\right)$ \\
\hline$\vartheta_{5}=\frac{\beta_{2}(x) \bar{X}}{2\left(\beta_{2}(x) \bar{X}+C_{x}\right)}$ & $\beta_{2}(x)$ & $C_{x}$ & $t_{C 1,5}=\bar{y}^{*}\left(v_{1}+v_{2} \frac{\bar{X}}{\bar{x}}\right) \exp$ & $\frac{\beta_{2}(x)(\bar{X}-\bar{x})}{\beta_{2}(x)(\bar{X}+\bar{x})+2 C_{x}}$ \\
\hline$\vartheta_{6}=\frac{C_{x} \bar{X}}{2\left(C_{x} \bar{X}+\beta_{2}(x)\right)}$ & $C_{x}$ & $\beta_{2}(x)$ & $t_{C 1,6}=\bar{y}^{*}\left(v_{1}+v_{2} \frac{\bar{X}}{\bar{x}}\right) \exp$ & $\frac{C_{x}(\bar{X}-\bar{x})}{C_{x}(\bar{X}+\bar{x})+2 \beta_{2}(x)}$ \\
\hline$\vartheta_{7}=\frac{C_{x} \bar{X}}{2\left(C_{x} \bar{X}+\rho\right)}$ & $C_{x}$ & $\rho$ & $t_{C 1,7}=\bar{y}^{*}\left(v_{1}+v_{2} \frac{\bar{X}}{\bar{x}}\right) \exp$ & $\left.\frac{C_{x}(\bar{X}-\bar{x})}{C_{x}(\bar{X}+\bar{x})+2 \rho}\right)$ \\
\hline$\vartheta_{8}=\frac{\rho \bar{X}}{2\left(\rho \bar{X}+C_{x}\right)}$ & $\rho$ & $C_{x}$ & $t_{C 1,8}=\bar{y}^{*}\left(v_{1}+v_{2} \frac{\bar{X}}{\bar{x}}\right) \exp$ & $\left.\frac{\rho(\bar{X}-\bar{x})}{\rho(\bar{X}+\bar{x})+2 C_{x}}\right)$ \\
\hline$\vartheta_{9}=\frac{\beta_{2}(x) \bar{X}}{2\left(\beta_{2}(x) \bar{X}+\rho\right)}$ & $\beta_{2}(x)$ & $\rho$ & $t_{C 1,9}=\bar{y}^{*}\left(v_{1}+v_{2} \frac{\bar{X}}{\bar{x}}\right) \exp$ & $\left.\frac{\beta_{2}(x)(\bar{X}-\bar{x})}{\beta_{2}(x)(\bar{X}+\bar{x})+2 \rho}\right)$ \\
\hline$\vartheta_{10}=\frac{\rho \bar{X}}{2\left(\rho \bar{X}+\beta_{2}(x)\right)}$ & $\rho$ & $\beta_{2}(x)$ & $t_{C 1,10}=\bar{y}^{*}\left(v_{1}+v_{2} \frac{\bar{X}}{\bar{x}}\right) \exp$ & $\frac{\rho(\bar{X}-\bar{x})}{\rho(\bar{X}+\bar{x})+2 \beta_{2}(x)}$ \\
\hline
\end{tabular}

We expand the right hand side of Equation (4) and after the terms, having powers of $e_{y}^{*}$ and $e_{x}$ two and higher, are neglected, we have

$$
\begin{aligned}
\left(t_{C 1, j}-\bar{Y}\right)=\bar{Y}\left(v_{1}-v_{1} \vartheta e_{x}+\frac{3 v_{1} \vartheta^{2}}{2}\right. & e_{x}^{2}+v_{2}-v_{2} \vartheta e_{x}+\frac{3 v_{2} \vartheta^{2}}{2} e_{x}^{2}-v_{2} e_{x}+v_{2} \vartheta e_{x}^{2} \\
& \left.+v_{2} e_{x}^{2}+v_{1} e_{y}^{*}-v_{1} \vartheta e_{y}^{*} e_{x}+v_{2} e_{y}^{*}-v_{2} \vartheta e_{y}^{*} e_{x}-v_{2} e_{y}^{*} e_{x}-1\right)
\end{aligned}
$$

We take the expectation on both sides of Equation (5) to derive $B\left(t_{C 1}\right)$ as

$$
\begin{aligned}
& E\left(t_{C 1, j}-\bar{Y}\right)=\bar{Y}\left(\left(v_{1}+v_{2}-1\right)+E\left(e_{x}^{2}\right)\left(\frac{3 \vartheta^{2}}{2}\left(v_{1}+v_{2}\right)+v_{2}(\vartheta+1)\right)-E\left(e_{y}^{*} e_{x}\right)\left(v_{1} \vartheta+v_{2}(\vartheta+1)\right)\right) \\
& B\left(t_{C 1, j}\right)=\bar{Y}\left(\left(v_{1}+v_{2}-1\right)+\lambda C_{x}^{2}\left(\frac{3 \vartheta^{2}}{2}\left(v_{1}+v_{2}\right)+v_{2}(\vartheta+1)\right)-\lambda C_{y x}\left(v_{1} \vartheta+v_{2}(\vartheta+1)\right)\right) .
\end{aligned}
$$

Expressions of the MSE are computed as follows: 


$$
\begin{aligned}
\left(t_{C 1, j}-\bar{Y}\right)^{2}=\bar{Y}^{2} & \left(1+v_{1}^{2}\left(1+e_{y}^{* 2}+4 \vartheta^{2} e_{x}^{2}-4 \vartheta e_{y}^{*} e_{x}\right)\right. \\
& +v_{2}^{2}\left(1+e_{y}^{*^{2}}+3 e_{x}^{2}+4 \vartheta e_{x}^{2}+4 \vartheta^{2} e_{x}^{2}-4 \vartheta e_{y}^{*} e_{x}-4 e_{y}^{*} e_{x}\right) \\
& +v_{1} v_{2}\left(2+2 e_{y}^{* 2}+2 e_{x}^{2}+4 \vartheta e_{x}^{2}+8 \vartheta^{2} e_{x}^{2}-8 \vartheta e_{y}^{*} e_{x}-4 e_{y}^{*} e_{x}\right) \\
& -v_{1}\left(2+3 \vartheta^{2} e_{x}^{2}-2 \vartheta e_{y}^{*} e_{x}\right) \\
& \left.-v_{2}\left(2+3 \vartheta^{2} e_{x}^{2}+2 \vartheta e_{x}^{2}+2 e_{x}^{2}-2 \vartheta e_{y}^{*} e_{x}-2 e_{y}^{*} e_{x}\right)\right), \\
E\left(t_{C 1, j}-\bar{Y}\right)^{2}= & \bar{Y}^{2}\left(1+v_{1}^{2}\left(1+E\left(e_{y}^{* 2}\right)+4 \vartheta^{2} E\left(e_{x}^{2}\right)-4 \vartheta E\left(e_{y}^{*} e_{x}\right)\right)\right. \\
& +v_{2}^{2}\left(1+E\left(e_{y}^{* 2}\right)+3 E\left(e_{x}^{2}\right)+4 \vartheta E\left(e_{x}^{2}\right)+4 \vartheta^{2} E\left(e_{x}^{2}\right)-4 \vartheta E\left(e_{y}^{*} e_{x}\right)-4 E\left(e_{y}^{*} e_{x}\right)\right) \\
& +v_{1} v_{2}\left(2+2 E\left(e_{y}^{* 2}\right)+2 E\left(e_{x}^{2}\right)+4 \vartheta E\left(e_{x}^{2}\right)+8 \vartheta^{2} E\left(e_{x}^{2}\right)-8 \vartheta E\left(e_{y}^{*} e_{x}\right)-4 E\left(e_{y}^{*} e_{x}\right)\right) \\
& -v_{1}\left(2+3 \vartheta^{2} E\left(e_{x}^{2}\right)-2 \vartheta E\left(e_{y}^{*} e_{x}\right)\right) \\
& \left.-v_{2}\left(2+3 \vartheta^{2} E\left(e_{x}^{2}\right)+2 \vartheta E\left(e_{x}^{2}\right)+2 E\left(e_{x}^{2}\right)-2 \vartheta E\left(e_{y}^{*} e_{x}\right)-2 E\left(e_{y}^{*} e_{x}\right)\right)\right),
\end{aligned}
$$

Where;

$$
\begin{aligned}
& A_{1}=\left(1+E\left(e_{y}^{*^{2}}\right)+4 \vartheta^{2} E\left(e_{x}^{2}\right)-4 \vartheta E\left(e_{y}^{*} e_{x}\right)\right) \\
& A_{2}=\left(1+E\left(e_{y}^{*^{2}}\right)+3 E\left(e_{x}^{2}\right)+4 \vartheta E\left(e_{x}^{2}\right)+4 \vartheta^{2} E\left(e_{x}^{2}\right)-4 \vartheta E\left(e_{y}^{*} e_{x}\right)-4 E\left(e_{y}^{*} e_{x}\right)\right) \\
& A_{3}=\left(1+3 / 2 \vartheta^{2} E\left(e_{x}^{2}\right)-\vartheta E\left(e_{y}^{*} e_{x}\right)\right) \\
& A_{4}=\left(1+3 / 2 \vartheta^{2} E\left(e_{x}^{2}\right)+\vartheta E\left(e_{x}^{2}\right)+E\left(e_{x}^{2}\right)-\vartheta E\left(e_{y}^{*} e_{x}\right)-E\left(e_{y}^{*} e_{x}\right)\right) \\
& A_{5}=\left(1+E\left(e_{y}^{* 2}\right)+E\left(e_{x}^{2}\right)+2 \vartheta E\left(e_{x}^{2}\right)+4 \vartheta^{2} E\left(e_{x}^{2}\right)-4 \vartheta E\left(e_{y}^{*} e_{x}\right)-2 E\left(e_{y}^{*} e_{x}\right)\right)
\end{aligned}
$$

and then we obtain $\operatorname{MSE}\left(t_{C 1, j}\right), j=1,2, \ldots, 10$ as

$$
\operatorname{MSE}\left(t_{C 1, j}\right)=\bar{Y}^{2}\left(1+A_{1} v_{1}^{2}+A_{2} v_{2}^{2}+2 A_{5} v_{1} v_{2}-2 A_{3} v_{1}-2 A_{4} v_{2}\right), j=1,2, \ldots, 10
$$

To obtain the minimum MSE of the $t_{C 1, j}, j=1,2, \ldots, 10$, we get the optimal values of $v_{1}$ and $v_{2}$, respectively, as follows: $v_{1}^{*}=\frac{A_{2} A_{3}-A_{5} A_{4}}{A_{1} A_{2}-A_{5}^{2}}, v_{2}^{*}=\frac{A_{1} A_{4}-A_{3} A_{5}}{A_{1} A_{2}-A_{5}^{2}}$.

Using the optimal values, $v_{1}^{*}$ and $v_{2}^{*}$, and substituting them in Equation (6), we obtain

$$
\begin{aligned}
& \operatorname{MSE}_{\text {min }}\left(t_{C 1, j}\right)=\bar{Y}^{2}\left(1+A_{1} v_{1}^{* 2}+A_{2} v_{2}^{* 2}+2 A_{5} v_{1}^{*} v_{2}^{*}-2 A_{3} v_{1}^{*}-2 A_{4} v_{2}^{*}\right) \\
& =\bar{Y}^{2}\left(1-\frac{A_{2} A_{3}^{2}+A_{1} A_{4}^{2}-2 A_{3} A_{4} A_{5}}{A_{1} A_{2}-A_{5}^{2}}\right), j=1,2, \ldots, 10
\end{aligned}
$$




\subsection{CASE II:}

The second family of estimators is proposed as

$t_{C 2, j}=\bar{y}^{*}\left(v_{1}+v_{2} \frac{\bar{X}}{\bar{x}^{*}}\right) \exp \left(\frac{\phi\left(\bar{X}-\bar{x}^{*}\right)}{\phi\left(\bar{X}+\bar{x}^{*}\right)+2 \varphi}\right), \mathrm{j}=1,2, \ldots, 10$.

Similarly, in the Case I, we re-write Equation (9) as

$$
\begin{aligned}
t_{C 2, j} & =\bar{Y}\left(1+e_{y}^{*}\right)\left(v_{1}+v_{2} \frac{\bar{X}}{\bar{X}\left(1+e_{x}^{*}\right)}\right)\left(1-\vartheta e_{x}^{*}+\frac{3 \vartheta^{2}}{2} e_{x}^{* 2}\right) \\
& =\bar{Y}\left(1+e_{y}^{*}\right)\left(v_{1}-v_{1} \vartheta e_{x}^{*}+\frac{3 v_{1} \vartheta^{2}}{2} e_{x}^{*^{2}}+v_{2}-v_{2} \vartheta e_{x}^{*}+\frac{3 v_{2} \vartheta^{2}}{2} e_{x}^{* 2}-v_{2} e_{x}^{*}+v_{2} \vartheta e_{x}^{* 2}+v_{2} e_{x}^{*^{2}}\right)
\end{aligned}
$$

We can write some members of the family of estimators for the Case II as in Table 4.

Expanding the right hand side of Equation (10) and then neglecting the terms having powers of $e_{y}^{*}$ and $e_{x}^{*}$ two and higher, we have

$$
\begin{aligned}
\left(t_{C 2, j}-\bar{Y}\right)=\bar{Y}\left(v_{1}-\right. & v_{1} \vartheta e_{x}^{*}+\frac{3 v_{1} \vartheta^{2}}{2} e_{x}^{*^{2}}+v_{2}-v_{2} \vartheta e_{x}^{*}+\frac{3 v_{2} \vartheta^{2}}{2} e_{x}^{* 2}-v_{2} e_{x}^{*}+v_{2} \vartheta e_{x}^{* 2} \\
& \left.+v_{2} e_{x}^{* 2}+v_{1} e_{y}^{*}-v_{1} \vartheta e_{y}^{*} e_{x}^{*}+v_{2} e_{y}^{*}-v_{2} \vartheta e_{y}^{*} e_{x}^{*}-v_{2} e_{y}^{*} e_{x}^{*}-1\right), j=1,2, \ldots, 10 .
\end{aligned}
$$

We take the expectation on both sides of Equation (11) as

$E\left(t_{C 2, j}-\bar{Y}\right)=\bar{Y}\left(\left(v_{1}+v_{2}-1\right)+E\left(e_{x}^{* 2}\right)\left(\frac{3 \vartheta^{2}}{2}\left(v_{1}+v_{2}\right)+v_{2}(\vartheta+1)\right)-E\left(e_{y}^{*} e_{x}^{*}\right)\left(v_{1} \vartheta+v_{2}(\vartheta+1)\right)\right)$

Using $E\left(e_{x}^{*}\right)=0, E\left(e_{y}^{*}\right)=0, E\left(e_{x}^{* 2}\right)=\lambda C_{x}^{2}+\frac{W_{2}(z-1)}{n} C_{x(2)}^{2}, E\left(e_{y}^{*} e_{x}^{*}\right)=\lambda \rho_{x y} C_{x} C_{y}+\frac{W_{2}(z-1)}{n} C_{x y(2)}$ $E\left(e_{y}^{* 2}\right)=\lambda C_{y}^{2}+\frac{W_{2}(z-1)}{n} C_{y(2)}^{2}$ notations, we obtain the bias of the $t_{C 2, j}, j=1,2, \ldots, 10$ as follows: 
Table 4- Some members of the $t_{C 2, j}, j=1,2, \ldots, 10$

\begin{tabular}{|c|c|c|c|c|}
\hline \multicolumn{3}{|c|}{ Values } & \multirow{2}{*}{\multicolumn{2}{|c|}{ Estimators }} \\
\hline$\vartheta_{i}$ & $\phi$ & $\varphi$ & & \\
\hline$\vartheta_{1}=\frac{\bar{X}}{2(\bar{X}+1)}$ & 1 & 1 & $t_{C 2,1}=\bar{y}^{*}\left(v_{1}+v_{2} \frac{\bar{X}}{\bar{x}^{*}}\right) \exp ($ & $\left.\frac{\left(\bar{X}-\bar{x}^{*}\right)}{\left(\bar{X}+\bar{x}^{*}\right)+2}\right)$ \\
\hline$\vartheta_{2}=\frac{\bar{X}}{2\left(\bar{X}+\beta_{2}(x)\right)}$ & 1 & $\beta_{2}(x)$ & $t_{C 2,2}=\bar{y}^{*}\left(v_{1}+v_{2} \frac{\bar{X}}{\bar{x}^{*}}\right) \exp$ & $\left(\frac{\left(\bar{X}-\bar{x}^{*}\right)}{\left(\bar{X}+\bar{x}^{*}\right)+2 \beta_{2}(x)}\right)$ \\
\hline$\vartheta_{3}=\frac{\bar{X}}{2\left(\bar{X}+C_{x}\right)}$ & 1 & $C_{x}$ & $t_{C 2,3}=\bar{y}^{*}\left(v_{1}+v_{2} \frac{\bar{X}}{\bar{x}^{*}}\right) \exp$ & $\left(\frac{\left(\bar{X}-\bar{x}^{*}\right)}{\left(\bar{X}+\bar{x}^{*}\right)+2 \varphi}\right)$ \\
\hline$\vartheta_{4}=\frac{\bar{X}}{2(\bar{X}+\rho)}$ & 1 & $\rho$ & $t_{C 2,4}=\bar{y}^{*}\left(v_{1}+v_{2} \frac{\bar{X}}{\bar{x}^{*}}\right) \exp$ & $\left(\frac{\left(\bar{X}-\bar{x}^{*}\right)}{\left(\bar{X}+\bar{x}^{*}\right)+2 \rho}\right)$ \\
\hline$\vartheta_{5}=\frac{\beta_{2}(x) \bar{X}}{2\left(\beta_{2}(x) \bar{X}+C_{x}\right)}$ & $\beta_{2}(x)$ & $C_{x}$ & $t_{C 2,5}=\bar{y}^{*}\left(v_{1}+v_{2} \frac{\bar{X}}{\bar{x}^{*}}\right) \exp$ & $\left(\frac{\beta_{2}(x)\left(\bar{X}-\bar{x}^{*}\right)}{\beta_{2}(x)\left(\bar{X}+\bar{x}^{*}\right)+2 C_{x}}\right.$ \\
\hline$\vartheta_{6}=\frac{C_{x} \bar{X}}{2\left(C_{x} \bar{X}+\beta_{2}(x)\right)}$ & $C_{x}$ & $\beta_{2}(x)$ & $t_{C 2,6}=\bar{y}^{*}\left(v_{1}+v_{2} \frac{\bar{X}}{\bar{x}^{*}}\right) \exp$ & $\left(\frac{C_{x}\left(\bar{X}-\bar{x}^{*}\right)}{C_{x}\left(\bar{X}+\bar{x}^{*}\right)+2 \beta_{2}(x)}\right.$ \\
\hline$\vartheta_{7}=\frac{C_{x} \bar{X}}{2\left(C_{x} \bar{X}+\rho\right)}$ & $C_{x}$ & $\rho$ & $t_{C 2,7}=\bar{y}^{*}\left(v_{1}+v_{2} \frac{\bar{X}}{\bar{x}^{*}}\right) \exp$ & $\left(\frac{C_{x}\left(\bar{X}-\bar{x}^{*}\right)}{C_{x}\left(\bar{X}+\bar{x}^{*}\right)+2 \rho}\right)$ \\
\hline$\vartheta_{8}=\frac{\rho \bar{X}}{2\left(\rho \bar{X}+C_{x}\right)}$ & $\rho$ & $C_{x}$ & $t_{C 2,8}=\bar{y}^{*}\left(v_{1}+v_{2} \frac{\bar{X}}{\bar{x}^{*}}\right) \exp$ & $\left(\frac{\rho\left(\bar{X}-\bar{x}^{*}\right)}{\rho\left(\bar{X}+\bar{x}^{*}\right)+2 C_{x}}\right)$ \\
\hline$\vartheta_{9}=\frac{\beta_{2}(x) \bar{X}}{2\left(\beta_{2}(x) \bar{X}+\rho\right)}$ & $\beta_{2}(x)$ & $\rho$ & $t_{C 2,9}=\bar{y}^{*}\left(v_{1}+v_{2} \frac{\bar{X}}{\bar{x}^{*}}\right) \exp$ & $\left(\frac{\beta_{2}(x)\left(\bar{X}-\bar{x}^{*}\right)}{\beta_{2}(x)\left(\bar{X}+\bar{x}^{*}\right)+2 \rho}\right)$ \\
\hline$\vartheta_{10}=\frac{\rho \bar{X}}{2\left(\rho \bar{X}+\beta_{2}(x)\right)}$ & $\rho$ & $\beta_{2}(x)$ & $t_{C 2,10}=\bar{y}^{*}\left(v_{1}+v_{2} \frac{\bar{X}}{\bar{x}^{*}}\right) \exp$ & $\left(\frac{\rho\left(\bar{X}-\bar{x}^{*}\right)}{\rho\left(\bar{X}+\bar{x}^{*}\right)+2 \beta_{2}(x)}\right)$ \\
\hline
\end{tabular}

$$
\begin{aligned}
B\left(t_{C 2, j}\right)=\bar{Y}\left(\left(v_{1}+v_{2}-1\right)+(\right. & \left.\lambda C_{x}^{2}+\frac{W_{2}(z-1)}{n} C_{x(2)}^{2}\right)\left(\frac{3 \vartheta^{2}}{2}\left(v_{1}+v_{2}\right)+v_{2}(\vartheta+1)\right) \\
& \left.-\left(\lambda C_{y x}+\frac{W_{2}(z-1)}{n} C_{y x(2)}\right)\left(v_{1} \vartheta+v_{2}(\vartheta+1)\right)\right), j=1,2, \ldots, 10 .
\end{aligned}
$$

Similarly, expressions of the $\operatorname{MSE}\left(t_{C 2, j}\right), j=1,2, \ldots, 10$ are computed, respectively, as follows:

$$
\begin{aligned}
\left(t_{C 2, j}-\bar{Y}\right)^{2}= & \bar{Y}^{2}\left(1+v_{1}^{2}\left(1+e_{y}^{* 2}+4 \vartheta^{2} e_{x}^{* 2}-4 \vartheta e_{y}^{*} e_{x}^{*}\right)\right. \\
& +v_{2}^{2}\left(1+e_{y}^{* 2}+3 e_{x}^{* 2}+4 \vartheta e_{x}^{* 2}+4 \vartheta^{2} e_{x}^{* 2}-4 \vartheta e_{y}^{*} e_{x}^{*}-4 e_{y}^{*} e_{x}^{*}\right) \\
& +v_{1} v_{2}\left(2+2 e_{y}^{* 2}+2 e_{x}^{* 2}+4 \vartheta e_{x}^{* 2}+8 \vartheta^{2} e_{x}^{* 2}-8 \vartheta e_{y}^{*} e_{x}^{*}-4 e_{y}^{*} e_{x}^{*}\right) \\
& -v_{1}\left(2+3 \vartheta^{2} e_{x}^{* 2}-2 \vartheta e_{y}^{*} e_{x}^{*}\right) \\
& \left.-v_{2}\left(2+3 \vartheta^{2} e_{x}^{* 2}+2 \vartheta e_{x}^{* 2}+2 e_{x}^{* 2}-2 \vartheta e_{y}^{*} e_{x}^{*}-2 e_{y}^{*} e_{x}^{*}\right)\right)
\end{aligned}
$$




$$
\begin{aligned}
E\left(t_{C 2, j}-\bar{Y}\right)^{2}= & \bar{Y}^{2}\left(1+v_{1}^{2}\left(1+E\left(e_{y}^{*^{2}}\right)+4 \vartheta^{2} E\left(e_{x}^{*^{2}}\right)-4 \vartheta E\left(e_{y}^{*} e_{x}^{*}\right)\right)\right. \\
& +v_{2}^{2}\left(1+E\left(e_{y}^{*^{2}}\right)+3 E\left(e_{x}^{*^{2}}\right)+4 \vartheta E\left(e_{x}^{*^{2}}\right)+4 \vartheta^{2} E\left(e_{x}^{*^{2}}\right)-4 \vartheta E\left(e_{y}^{*} e_{x}^{*}\right)-4 E\left(e_{y}^{*} e_{x}^{*}\right)\right) \\
& +v_{1} v_{2}\left(2+2 E\left(e_{y}^{*^{2}}\right)+2 E\left(e_{x}^{*^{2}}\right)+4 \vartheta E\left(e_{x}^{*^{2}}\right)+8 \vartheta^{2} E\left(e_{x}^{*^{2}}\right)-8 \vartheta E\left(e_{y}^{*} e_{x}^{*}\right)-4 E\left(e_{y}^{*} e_{x}^{*}\right)\right) \\
& -v_{1}\left(2+3 \vartheta^{2} E\left(e_{x}^{*^{2}}\right)-2 \vartheta E\left(e_{y}^{*} e_{x}^{*}\right)\right) \\
& \left.-v_{2}\left(2+3 \vartheta^{2} E\left(e_{x}^{*^{2}}\right)+2 \vartheta E\left(e_{x}^{* 2}\right)+2 E\left(e_{x}^{* 2}\right)-2 \vartheta E\left(e_{y}^{*} e_{x}^{*}\right)-2 E\left(e_{y}^{*} e_{x}^{*}\right)\right)\right)
\end{aligned}
$$

Where;

$$
\begin{aligned}
& B_{1}=\left(1+E\left(e_{y}^{*^{2}}\right)+4 \vartheta^{2} E\left(e_{x}^{*^{2}}\right)-4 \vartheta E\left(e_{y}^{*} e_{x}^{*}\right)\right) \\
& B_{2}=\left(1+E\left(e_{y}^{*^{2}}\right)+3 E\left(e_{x}^{*^{2}}\right)+4 \vartheta E\left(e_{x}^{*^{2}}\right)+4 \vartheta^{2} E\left(e_{x}^{*^{2}}\right)-4 \vartheta E\left(e_{y}^{*} e_{x}^{*}\right)-4 E\left(e_{y}^{*} e_{x}^{*}\right)\right) \\
& B_{3}=\left(1+3 / 2 \vartheta^{2} E\left(e_{x}^{*^{2}}\right)-\vartheta E\left(e_{y}^{*} e_{x}^{*}\right)\right) \\
& B_{4}=\left(1+3 / 2 \vartheta^{2} E\left(e_{x}^{* 2}\right)+\vartheta E\left(e_{x}^{* 2}\right)+E\left(e_{x}^{*^{2}}\right)-\vartheta E\left(e_{y}^{*} e_{x}^{*}\right)-E\left(e_{y}^{*} e_{x}^{*}\right)\right) \\
& B_{5}=\left(1+E\left(e_{y}^{*^{2}}\right)+E\left(e_{x}^{*^{2}}\right)+2 \vartheta E\left(e_{x}^{*^{2}}\right)+4 \vartheta^{2} E\left(e_{x}^{*^{2}}\right)-4 \vartheta E\left(e_{y}^{*} e_{x}^{*}\right)-2 E\left(e_{y}^{*} e_{x}^{*}\right)\right)
\end{aligned}
$$

and then we obtain $\operatorname{MSE}\left(t_{C 2, j}\right), j=1,2, \ldots, 10$ as

$$
\operatorname{MSE}\left(t_{C 2, j}\right)=\bar{Y}^{2}\left(1+B_{1} v_{1}^{2}+B_{2} v_{2}^{2}+2 B_{5} v_{1} v_{2}-2 B_{3} v_{1}-2 B_{4} v_{2}\right), j=1,2, \ldots, 10
$$

The optimal values of $v_{1}$ and $v_{2}$ are obtained as

$$
v_{1}^{* *}=\frac{B_{2} B_{3}-B_{5} B_{4}}{B_{1} B_{2}-B_{5}^{2}}, v_{2}^{* *}=\frac{B_{1} B_{4}-B_{3} B_{5}}{B_{1} B_{2}-B_{5}^{2}}
$$

Using $v_{1}^{* *}$ and $v_{2}^{* *}$ and substituting them in Equation (22), we get

$$
\begin{gathered}
M S E_{\min }\left(t_{C 2, j}\right)=\bar{Y}^{2}\left(1+B_{1} v_{1}^{* * 2}+B_{2} v_{2}^{* * 2}+2 B_{5} v_{1}^{* *} v_{2}^{* *}-2 B_{3} v_{1}^{* *}-2 B_{4} v_{2}^{* *}\right) \\
=\bar{Y}^{2}\left(1-\frac{B_{2} B_{3}^{2}+B_{1} B_{4}^{2}-2 B_{3} B_{4} B_{5}}{B_{1} B_{2}-B_{5}^{2}}\right), j=1,2, \ldots, 10 .
\end{gathered}
$$

\section{Efficiency Comparisons}

One of the important features of an estimator is efficiency. After the theoretical inferences of the $t_{C 1, j}$ and $t_{C 2, j} j=1,2, \ldots, 10$ estimators, we obtain the efficiency comparisons for each case, separately. The conditions are given for the Case I and Case II in the next sub-sections.

\section{a. Efficiency comparisons for the first case}

We use the variance of the $t_{H}$ and the MSE Equation of the $t_{R 1}, t_{\exp 1}, t_{r e g 1}, t_{C 1, j}, j=1,2, \ldots, 10$, respectively, as follows: 
$\operatorname{MSE}\left(t_{R 1}\right)=\bar{Y}^{2}\left(\lambda\left(C_{x}^{2}-2 C_{y x}+C_{y}^{2}\right)+\frac{W_{2}(z-1)}{n} C_{y(2)}^{2}\right)$,
$\operatorname{MSE}\left(t_{\text {exp1 }}\right)=\bar{Y}^{2}\left(\lambda\left(C_{y}^{2}+\frac{C_{x}^{2}}{4}-C_{y x}\right)+\frac{W_{2}(z-1)}{n} C_{y(2)}^{2}\right)$,

$\operatorname{MSE}\left(t_{\text {reg1 } 1}\right)=\bar{Y}^{2}\left(\lambda C_{y}^{2}\left(1-\rho_{x y}^{2}\right)+\frac{W_{2}(z-1)}{n} C_{y(2)}^{2}\right)$

and using Equations (2), (14), (15), and (16), we obtain that

- $\left[V\left(t_{H}\right)-M S E_{\min }\left(t_{C 1, j}\right)\right]>0, j=1,2, \ldots, 10$

$$
\left(\lambda C_{y}^{2}+\frac{W_{2}(z-1)}{n} C_{y(2)}^{2}\right)-\left(1-\frac{A_{2} A_{3}^{2}+A_{1} A_{4}^{2}-2 A_{3} A_{4} A_{5}}{A_{1} A_{2}-A_{5}^{2}}\right)>0
$$

- $\left[\operatorname{MSE}\left(t_{R 1}\right)-M S E_{\min }\left(t_{C 1, j}\right)\right]>0, j=1,2, \ldots, 10$

$$
\left(\lambda\left(C_{x}^{2}-2 C_{y x}+C_{y}^{2}\right)+\frac{W_{2}(z-1)}{n} C_{y(2)}^{2}\right)-\left(1-\frac{A_{2} A_{3}^{2}+A_{1} A_{4}^{2}-2 A_{3} A_{4} A_{5}}{A_{1} A_{2}-A_{5}^{2}}\right)>0
$$

- $\left[\operatorname{MSE}\left(t_{\exp 1}\right)-M S E_{\min }\left(t_{C 1, j}\right)\right]>0, j=1,2, \ldots, 10$

$$
\left(\lambda\left(C_{y}^{2}+\frac{C_{x}^{2}}{4}-C_{y x}\right)+\frac{W_{2}(z-1)}{n} C_{y(2)}^{2}\right)-\left(1-\frac{A_{2} A_{3}^{2}+A_{1} A_{4}^{2}-2 A_{3} A_{4} A_{5}}{A_{1} A_{2}-A_{5}^{2}}\right)>0
$$

- $\left[\operatorname{MSE}\left(t_{\text {reg } 1}\right)-M S E_{\min }\left(t_{C 1, j}\right)\right]>0, j=1,2, \ldots, 10$

$$
\left(\lambda C_{y}^{2}\left(1-\rho_{x y}^{2}\right)+\frac{W_{2}(z-1)}{n} C_{y(2)}^{2}\right)-\left(1-\frac{A_{2} A_{3}^{2}+A_{1} A_{4}^{2}-2 A_{3} A_{4} A_{5}}{A_{1} A_{2}-A_{5}^{2}}\right)>0
$$

\section{b. Efficiency comparisons for the second case}

We use the variance of the $t_{H}$ and the MSE Equation of the $t_{R 2}, t_{\exp 2}, t_{r e g 2}, t_{C 2, j}, j=1,2, \ldots, 10$, respectively, as follows:

$$
\begin{aligned}
& \operatorname{MSE}\left(t_{R 2}\right)=\bar{Y}^{2}\left(\lambda\left(C_{x}^{2}-2 C_{y x}+C_{y}^{2}\right)+\frac{W_{2}(z-1)}{n}\left(C_{y(2)}^{2}+C_{x(2)}^{2}-2 C_{y x(2)}\right)\right) \\
& \operatorname{MSE}\left(t_{\exp 2}\right)=\bar{Y}^{2}\left(\lambda C_{y}^{2}+\lambda \frac{C_{x}^{2}}{4}-\lambda C_{y x}+\frac{W_{2}(z-1)}{n}\left(C_{y(2)}^{2}+\frac{C_{x(2)}^{2}}{4}-C_{y x(2)}\right)\right) \\
& \operatorname{MSE}\left(t_{\text {reg } 2}\right)=\bar{Y}^{2}\left(\lambda C_{y}^{2}\left(1-\rho_{x y}^{2}\right)+\frac{W_{2}(z-1)}{n}\left(C_{y(2)}^{2}+\rho_{x y}^{2} \frac{C_{y}^{2}}{C_{x}^{2}} C_{x(2)}^{2}-2 \rho_{x y} \frac{C_{y}}{C_{x}} C_{y x(2)}\right)\right),
\end{aligned}
$$

and using Equations (2), (21), (22) and (23), we obtain that

$$
\begin{aligned}
\cdot & {\left[V\left(t_{H}\right)-M S E_{\min }\left(t_{C 2, j}\right)\right]>0, j=1,2, \ldots, 10 } \\
& \left(\lambda C_{y}^{2}+\frac{W_{2}(z-1)}{n} C_{y(2)}^{2}\right)-\left(1-\frac{B_{2} B_{3}^{2}+B_{1} B_{4}^{2}-2 B_{3} B_{4} B_{5}}{B_{1} B_{2}-B_{5}^{2}}\right)>0 \\
\cdot & {\left[M S E\left(t_{R 2}\right)-M S E_{\min }\left(t_{C 2, j}\right)\right]>0, j=1,2, \ldots, 10 } \\
& \left(\lambda\left(C_{x}^{2}-2 C_{y x}+C_{y}^{2}\right)+\frac{W_{2}(z-1)}{n}\left(C_{y(2)}^{2}+C_{x(2)}^{2}-2 C_{y x(2)}\right)\right)-\left(1-\frac{B_{2} B_{3}^{2}+B_{1} B_{4}^{2}-2 B_{3} B_{4} B_{5}}{B_{1} B_{2}-B_{5}^{2}}\right)>0
\end{aligned}
$$


- $\left[\operatorname{MSE}\left(t_{\exp 2}\right)-M S E_{\min }\left(t_{C 2, j}\right)\right]>0, j=1,2, \ldots, 10$

$\left(\lambda C_{y}^{2}+\lambda \frac{C_{x}^{2}}{4}-\lambda C_{y x}+\frac{W_{2}(z-1)}{n}\left(C_{y(2)}^{2}+\frac{C_{x(2)}^{2}}{4}-C_{y x(2)}\right)\right)-\left(1-\frac{B_{2} B_{3}^{2}+B_{1} B_{4}^{2}-2 B_{3} B_{4} B_{5}}{B_{1} B_{2}-B_{5}^{2}}\right)>0$

- $\left[\operatorname{MSE}\left(t_{\text {reg } 2}\right)-M S E_{\min }\left(t_{C 2, j}\right)\right]>0, j=1,2, \ldots, 10$

$\left(\lambda C_{y}^{2}\left(1-\rho_{x y}^{2}\right)+\frac{W_{2}(z-1)}{n}\left(C_{y(2)}^{2}+\rho_{x y}^{2} \frac{C_{y}^{2}}{C_{x}^{2}} C_{x(2)}^{2}-2 \rho_{x y} \frac{C_{y}}{C_{x}} C_{y x(2)}\right)\right)-\left(1-\frac{B_{2} B_{3}^{2}+B_{1} B_{4}^{2}-2 B_{3} B_{4} B_{5}}{B_{1} B_{2}-B_{5}^{2}}\right)>0$

The family of estimators, $t_{C 1, j}$ and $t_{C 2, j} \mathrm{j}=1,2, \ldots, 10$ are more efficient than others if the conditions for the efficiency, given in Equation (17) - Equation (20) and Equation (24) - Equation (27), are satisfied for both cases, respectively.

\section{Empirical Studies}

After theoretical inferences and comparisons, numerical illustrations are conducted in this section. The two real data sets are used to deal with the situation in which $t_{C 1, j}$ and $t_{C 2, j}, j=1,2, \ldots, 10$ estimators are more efficient than others. These data sets are also related with the agriculture for the purpose of showing the appropriateness of the proposed estimators, $t_{C 1, j}$ and $t_{C 2, j}, j=1,2, \ldots, 10$, in the agricultural field.

The parameter values for the Population 1 and Population 2 are given as follows:

Population 1. [Khare and Sinha (2009)]

In this population, the number of agriculture labors and the area of the village (in hectares) are considered as $y$ and $x$, respectively. In this population, $25 \%$ of villages is greater than 160 ha and they symbolize the non-response group.

\begin{tabular}{ccccc}
\hline$N=96$ & $n=40$ & $\rho_{y x(2)}=0.72$ & $C_{y x}=0.8232$ & $C_{y x(2)}=1.4077$ \\
$\bar{X}=144.87$ & $W_{2}=0.25$ & $\rho_{y x}=0.77$ & $C_{x}=0.81$ & $C_{x(2)}=0.94$ \\
$\bar{Y}=137.92$ & $\lambda=0.01458, f=0.42$ & $\beta_{2}(x)=1.19$ & $C_{y}=1.32$ & $C_{y(2)}=2.08$ \\
\hline
\end{tabular}

Population 2. [Khare and Srivastava (1993)]

In population 2, the cultivated area (in acres) and the population of the village are considered as $y$ and $x$, respectively. In this population, 14 villages (i.e. $20 \%$ villages) are considered as non-response units.

\begin{tabular}{ccccc}
\hline$N=70$ & $n=35$ & $\rho_{y x(2)}=0.445$ & $C_{y x}=0.3896$ & $C_{y x(2)}=0.104$ \\
$\bar{X}=1755.53$ & $W_{2}=0.2$ & $\rho_{y x}=0.778$ & $C_{x}=0.801$ & $C_{x(2)}=0.574$ \\
$\bar{Y}=981.29$ & $\lambda=0.0143, f=0.50$ & $\beta_{2}(x)=0.34$ & $C_{y}=0.6254$ & $C_{y(2)}=0.4087$ \\
\hline
\end{tabular}

In Tables 5 and 6 , we present the MSE values of the $t_{C 1, j}, j=1,2, \ldots, 10$ and some main estimators for the Populations 1 and 2 , respectively. According to the results, we conclude that the $t_{C 1, j}, j=1,2, \ldots, 10$ estimator has the minimum MSE value among other compared estimators using the different values of $z$ for both populations.

Similarly, we present the MSE values of the $t_{C 2, j}, j=1,2, \ldots, 10$ and other same estimators for the Populations 1 and 2, respectively, in Tables 7 and 8 . According to the various values of $z$, we conclude that the $t_{C 2, j}, j=1,2, \ldots, 10$ estimator has the minimum MSE value among others for both populations under the Case II as in the Case I. 
Table 5- MSE values of the $t_{C 1, j}, j=1,2, \ldots, 10$ and existing estimators for the Population I

\begin{tabular}{|c|c|c|c|c|c|}
\hline Estimators & $z=2$ & $z=3$ & $z=4$ & $z=5$ & $z=6$ \\
\hline$t_{C 1,1}$ & 686.3603 & 1152.707 & 1595.907 & 2017.642 & 2419.43350 \\
\hline$t_{C 1,2}$ & 686.3668 & 1152.718 & 1595.922 & 2017.660 & 2419.45502 \\
\hline$t_{C 1,3}$ & 686.3542 & 1152.697 & 1595.893 & 2017.624 & 2419.41298 \\
\hline$t_{C 1,4}$ & 686.3529 & 1152.695 & 1595.890 & 2017.621 & 2419.40865 \\
\hline$t_{C 1,5}$ & 686.3498 & 1152.690 & 1595.883 & 2017.612 & 2419.39836 \\
\hline$t_{C 1,6}$ & 686.3758 & 1152.733 & 1595.942 & 2017.685 & 2419.48517 \\
\hline$t_{C 1,7}$ & 686.3587 & 1152.704 & 1595.903 & 2017.637 & 2419.42817 \\
\hline$t_{C 1,8}$ & 686.3620 & 1152.710 & 1595.911 & 2017.646 & 2419.43910 \\
\hline$t_{C 1,9}$ & 686.3487 & 1152.688 & 1595.881 & 2017.609 & 2419.39474 \\
\hline$t_{C 1,10}$ & 686.3782 & 1152.737 & 1595.948 & 2017.692 & 2419.49339 \\
\hline$t_{H}$ & 997.7000 & 1512.053 & 2026.406 & 2540.759 & 3055.11160 \\
\hline$t_{R 1}$ & 722.9411 & 1237.294 & 1751.647 & 2265.999 & 2780.35271 \\
\hline$t_{r e g 1}$ & 711.1235 & 1225.476 & 1739.829 & 2254.182 & 2768.53507 \\
\hline$t_{\exp 1}$ & 814.8195 & 1329.172 & 1843.525 & 2357.878 & 2872.23111 \\
\hline
\end{tabular}

Table 6- MSE values of the $t_{C 1, j}, j=1,2, \ldots, 10$ and existing estimators for the Population II

\begin{tabular}{|c|c|c|c|c|c|}
\hline Estimators & $z=2$ & $z=3$ & $z=4$ & $z=5$ & $z=6$ \\
\hline$t_{C 1,1}$ & 3021.606751 & 3931.230876 & 4839.120003 & 5745.279089 & 6649.71308 \\
\hline$t_{C 1,2}$ & 3021.605021 & 3931.228783 & 4839.117541 & 5745.276256 & 6649.70987 \\
\hline$t_{C 1,3}$ & 3021.60623 & 3931.230246 & 4839.119261 & 5745.278236 & 6649.71211 \\
\hline$t_{C 1,4}$ & 3021.60617 & 3931.230173 & 4839.119176 & 5745.278138 & 6649.71200 \\
\hline$t_{C 1,5}$ & 3021.61030 & 3931.235167 & 4839.125048 & 5745.284896 & 6649.71965 \\
\hline$t_{C 1,6}$ & 3021.60524 & 3931.229051 & 4839.117856 & 5745.276618 & 6649.71028 \\
\hline$t_{C 1,7}$ & 3021.60668 & 3931.230786 & 4839.119896 & 5745.278967 & 6649.71294 \\
\hline$t_{C 1,8}$ & 3021.60683 & 3931.230969 & 4839.120112 & 5745.279215 & 6649.71322 \\
\hline$t_{C 1,9}$ & 3021.61012 & 3931.234955 & 4839.124798 & 5745.284609 & 6649.71933 \\
\hline$t_{C 1,10}$ & 3021.60528 & 3931.229090 & 4839.117903 & 5745.276672 & 6649.71034 \\
\hline$t_{H}$ & 6299.48072 & 7218.587508 & 8137.694294 & 9056.801081 & 9975.90787 \\
\hline$t_{R 1}$ & 4402.05564 & 5321.162427 & 6240.269214 & 7159.3760 & 8078.48279 \\
\hline$t_{\text {reg1 }}$ & 3042.82647 & 3961.933251 & 4881.040038 & 5800.146825 & 6719.25361 \\
\hline$t_{\exp 1}$ & 3144.83018 & 4063.936966 & 4983.043753 & 5902.15054 & 6821.25733 \\
\hline
\end{tabular}

Table 7- MSE values of the $t_{C 2, j}, j=1,2, \ldots, 10$ and existing estimators for the Population I

\begin{tabular}{|c|c|c|c|c|c|}
\hline Estimators & $z=2$ & $z=3$ & $z=4$ & $z=5$ & $z=6$ \\
\hline$t_{C 2,1}$ & 444.1827 & 685.4467 & 921.5507 & 1153.376 & 1381.321333 \\
\hline$t_{C 3,2}$ & 444.1895 & 685.4614 & 921.5759 & 1153.414 & 1381.37485 \\
\hline$t_{C 2,3}$ & 444.1762 & 685.4328 & 921.5268 & 1153.339 & 1381.270296 \\
\hline$t_{C 2,4}$ & 444.1748 & 685.4298 & 921.5217 & 1153.332 & 1381.259528 \\
\hline$t_{C 3,5}$ & 444.1716 & 685.4228 & 921.5097 & 1153.314 & 1381.233925 \\
\hline$t_{C 2,6}$ & 444.199 & 685.4819 & 921.6111 & 1153.467 & 1381.449836 \\
\hline$t_{C 2,7}$ & 444.181 & 685.4431 & 921.5445 & 1153.366 & 1381.308086 \\
\hline$t_{C 2,8}$ & 444.1844 & 685.4505 & 921.5573 & 1153.386 & 1381.335255 \\
\hline$t_{C 2,9}$ & 444.1704 & 685.4204 & 921.5054 & 1153.307 & 1381.224929 \\
\hline$t_{C 2,10}$ & 444.2016 & 685.4875 & 921.6207 & 1153.482 & 1381.470268 \\
\hline$t_{H}$ & 997.7000 & 1512.053 & 2026.4058 & 2540.7587 & 3055.111599 \\
\hline$t_{R 2}$ & 493.2647 & 777.9412 & 1062.6176 & 1347.2940 & 1631.970482 \\
\hline$t_{\text {reg2 }}$ & 456.5109 & 716.2512 & 975.9915 & 1235.732 & 1495.472056 \\
\hline$t_{\operatorname{exp2}}$ & 673.7192 & 1046.972 & 1420.2242 & 1793.4767 & 2166.729263 \\
\hline
\end{tabular}


Table 8- MSE values of the $t_{C 2, j}, j=1,2, \ldots, 10$ and existing estimators for the Population II

\begin{tabular}{|c|c|c|c|c|c|}
\hline Estimators & $z=2$ & $z=3$ & $z=4$ & $z=5$ & $z=6$ \\
\hline$t_{C 2,1}$ & 2965.84881 & 3783.26051 & 4576.61636 & 5353.6645 & 6119.0051 \\
\hline$t_{C 3,2}$ & 2965.84763 & 3783.25975 & 4576.61625 & 5353.6652 & 6119.0068 \\
\hline$t_{C 2,3}$ & 2965.84846 & 3783.26028 & 4576.61633 & 5353.6647 & 6119.0055 \\
\hline$t_{C 2,4}$ & 2965.84842 & 3783.26026 & 4576.61633 & 5353.6647 & 6119.0056 \\
\hline$t_{C 3,5}$ & 2965.85124 & 3783.26205 & 4576.61658 & 5353.6630 & 6119.0014 \\
\hline$t_{C 2,6}$ & 2965.84778 & 3783.25985 & 4576.61626 & 5353.6651 & 6119.0065 \\
\hline$t_{C 2,8}$ & 2965.84887 & 3783.26055 & 4576.61637 & 5353.6644 & 6119.0049 \\
\hline$t_{C 2,9}$ & 2965.85112 & 3783.26198 & 4576.61657 & 5353.6630 & 6119.0016 \\
\hline$t_{C 2,10}$ & 2965.84780 & 3783.25987 & 4576.61627 & 5353.6651 & 6119.0065 \\
\hline$t_{H}$ & 6299.48072 & 7218.58751 & 8137.69429 & 9056.8011 & 9975.9079 \\
\hline$t_{R 2}$ & 5065.70210 & 6648.45535 & 8231.20860 & 9813.9618 & 11396.7150 \\
\hline$t_{\text {reg2 }}$ & 3013.88002 & 3904.04037 & 4794.20072 & 5684.3611 & 6574.52141 \\
\hline$t_{\text {exp2 }}$ & 3023.57939 & 3821.43539 & 4619.29140 & 5417.1474 & 6215.0034 \\
\hline
\end{tabular}

The Percentage Relative Efficiency (PRE) values of $t_{C 1, j}, j=1,2, \ldots, 10$ and $t_{C 2, j}, j=1,2, \ldots, 10$ and existing estimators in literature for various values of $z$ with respect to the Hansen and Hurwitz estimator $\left(t_{H}\right)$ are presented in Tables $9-12$ based on Populations $1-2$, respectively, using the formulae as follows:

$$
\operatorname{PRE}(t)=\frac{\operatorname{MSE}\left(t_{H}\right)}{\operatorname{MSE}(t)} x 100
$$

Table 9- PRE values of the $t_{C 1, j}, j=1,2, \ldots, 10$ and existing estimators for the Population I

\begin{tabular}{|c|c|c|c|c|c|}
\hline Estimators & $z=2$ & $z=3$ & $z=4$ & $z=5$ & $z=6$ \\
\hline$t_{C 1,1}$ & 145.3609723 & 131.1740864 & 126.9751881 & 125.9271596 & 126.2738405 \\
\hline$t_{C 1,2}$ & 145.3596104 & 131.1728691 & 126.9740256 & 125.9260232 & 126.2727173 \\
\hline$t_{C 1,3}$ & 145.3622712 & 131.1752472 & 126.9762967 & 125.9282433 & 126.2749115 \\
\hline$t_{C 1,4}$ & 145.3625452 & 131.1754922 & 126.9765306 & 125.928472 & 126.2751375 \\
\hline$t_{C 1,5}$ & 145.3631968 & 131.1760745 & 126.9770867 & 125.9290156 & 126.2756748 \\
\hline$t_{C 1,6}$ & 145.3577021 & 131.1711634 & 126.9723966 & 125.9244308 & 126.2711436 \\
\hline$t_{C 1,7}$ & 145.3613094 & 131.1743877 & 126.9754758 & 125.9274409 & 126.2741185 \\
\hline$t_{C 1,8}$ & 145.360618 & 131.1737697 & 126.9748857 & 125.926864 & 126.2735483 \\
\hline$t_{C 1,9}$ & 145.3634257 & 131.1762791 & 126.9772821 & 125.9292066 & 126.2758636 \\
\hline$t_{C 1,10}$ & 145.3571821 & 131.1706986 & 126.9719528 & 125.9239969 & 126.2707148 \\
\hline$t_{H}$ & 100 & 100 & 100 & 100 & 100 \\
\hline$t_{R 1}$ & 138.0057057 & 122.2064342 & 115.6857459 & 112.1252828 & 109.8821594 \\
\hline$t_{r e g 1}$ & 140.2991209 & 123.3849076 & 116.4715312 & 112.713104 & 110.3511972 \\
\hline$t_{\exp 1}$ & 122.4442927 & 113.7589736 & 109.9201504 & 107.7561464 & 106.3671926 \\
\hline
\end{tabular}

Table 10- PRE values of the $t_{C 1, j}, j=1,2, \ldots, 10$ and existing estimators for the Population II

\begin{tabular}{cccccc}
\hline Estimators & $z=2$ & $z=3$ & $z=4$ & $z=5$ & $z=6$ \\
\hline$t_{C 1,1}$ & 208.4811572 & 183.6215612 & 168.164755 & 157.6390101 & 150.020125 \\
$t_{C 1,2}$ & 208.4812766 & 183.6216589 & 168.1648405 & 157.6390878 & 150.0201974 \\
$t_{C 1,3}$ & 208.4811932 & 183.6215906 & 168.1647807 & 157.6390335 & 150.0201468 \\
$t_{C 1,4}$ & 208.4811973 & 183.621594 & 168.1647837 & 157.6390362 & 150.0201493 \\
$t_{C 1,5}$ & 208.4809126 & 183.6213607 & 168.1645796 & 157.6388507 & 150.0199766 \\
$t_{C 1,6}$ & 208.4812613 & 183.6216464 & 168.1648295 & 157.6390778 & 150.0201881 \\
$t_{C 1,7}$ & 208.4811624 & 183.6215654 & 168.1647587 & 157.6390134 & 150.0201281 \\
$t_{C 1,8}$ & 208.4811519 & 183.6215568 & 168.1647511 & 157.6390066 & 150.0201218 \\
$t_{C 1,9}$ & 208.4809247 & 183.6213707 & 168.1645883 & 157.6388586 & 150.0199839 \\
$t_{C 1,10}$ & 208.481259 & 183.6216446 & 168.1648279 & 157.6390764 & 150.0201867 \\
$t_{H}$ & 100 & 100 & 100 & 100 & 100 \\
$t_{R 1}$ & 143.1031599 & 135.6580936 & 130.4061414 & 126.50266 & 123.487394 \\
$t_{r e g 1}$ & 207.0272753 & 182.1986149 & 166.7204987 & 156.14779 & 148.4675002 \\
$t_{\exp 1}$ & 200.31227 & 177.6254791 & 163.307703 & 153.4491711 & 146.2473469 \\
\hline
\end{tabular}


According to the PRE values, the proposed family of estimators, $t_{C 1, j}, j=1,2, \ldots, 10$, perform better than compared estimators, $t_{R 1}, t_{r e g 1}$ and $t_{\exp 1}$, under the Case I for both populations.

Table 11- PRE values of the $t_{C 2, j}, j=1,2, \ldots, 10$ and existing estimators for the Population I

\begin{tabular}{cccccc}
\hline Estimators & $z=2$ & $z=3$ & $z=4$ & $z=5$ & $z=6$ \\
\hline$t_{C 2,1}$ & 224.6148091 & 220.5937908 & 219.8908604 & 220.2888855 & 221.1731279 \\
$t_{C 3,2}$ & 224.6113763 & 220.5890807 & 219.8848622 & 220.2816 & 221.1645593 \\
$t_{C 2,3}$ & 224.6180833 & 220.5982832 & 219.8965812 & 220.2958339 & 221.1813001 \\
$t_{C 2,4}$ & 224.6187742 & 220.5992311 & 219.8977883 & 220.2973 & 221.1830244 \\
$t_{C 3,5}$ & 224.6204171 & 220.6014849 & 219.9006584 & 220.300786 & 221.1871243 \\
$t_{C 2,6}$ & 224.6065675 & 220.582482 & 219.8764587 & 220.2713929 & 221.1525544 \\
$t_{C 2,7}$ & 224.6156589 & 220.5949568 & 219.8923452 & 220.290689 & 221.175249 \\
$t_{C 2,8}$ & 224.613916 & 220.5925654 & 219.8892999 & 220.2869901 & 221.1708987 \\
$t_{C 2,9}$ & 224.6209943 & 220.6022769 & 219.9016669 & 220.3020109 & 221.188565 \\
$t_{C 2,10}$ & 224.6052573 & 220.5806842 & 219.8741691 & 220.2686119 & 221.1492835 \\
$t_{H}$ & 100 & 100 & 100 & 100 & 100 \\
$t_{R 2}$ & 202.2646312 & 194.3659785 & 190.6994416 & 188.5823463 & 187.2038515 \\
$t_{r e g 2}$ & 218.5490019 & 211.1065133 & 207.6253587 & 205.6076222 & 204.2907847 \\
$t_{\text {exp2 }}$ & 148.0884119 & 144.421566 & 142.6821051 & 141.6666661 & 141.0010771 \\
\hline
\end{tabular}

Table 12- PRE values of the $t_{C 2, j}, j=1,2, \ldots, 10$ and existing estimators for the Population II

\begin{tabular}{cccccc}
\hline Estimators & $z=2$ & $z=3$ & $z=4$ & $z=5$ & $z=6$ \\
\hline$t_{C 2,1}$ & 212.4006015 & 190.8033424 & 177.8102783 & 169.1701289 & 163.0315363 \\
$t_{C 3,2}$ & 212.4006865 & 190.8033807 & 177.8102828 & 169.170106 & 163.0314897 \\
$t_{C 2,3}$ & 212.4006271 & 190.8033539 & 177.8102797 & 169.170122 & 163.0315222 \\
$t_{C 2,4}$ & 212.40063 & 190.8033552 & 177.8102798 & 169.1701212 & 163.0315206 \\
$t_{C 3,5}$ & 212.4004277 & 190.8032646 & 177.8102699 & 169.1701767 & 163.0316328 \\
$t_{C 2,6}$ & 212.4006756 & 190.8033758 & 177.8102822 & 169.1701089 & 163.0314957 \\
$t_{C 2,7}$ & 212.4006052 & 190.8033441 & 177.8102785 & 169.1701279 & 163.0315342 \\
$t_{C 2,8}$ & 212.4005977 & 190.8033407 & 177.8102781 & 169.1701299 & 163.0315383 \\
$t_{C 2,9}$ & 212.4004363 & 190.8032685 & 177.8102703 & 169.1701743 & 163.031628 \\
$t_{C 2,10}$ & 212.400674 & 190.803375 & 177.8102822 & 169.1701094 & 163.0314965 \\
$t_{H}$ & 100 & 100 & 100 & 100 & 100 \\
$t_{R 2}$ & 124.3555305 & 108.5754078 & 98.86390559 & 92.28486137 & 87.53318644 \\
$t_{r e g 2}$ & 209.0156433 & 184.9004319 & 169.7403754 & 159.3283921 & 151.7358793 \\
$t_{\operatorname{exp2}}$ & 208.34514 & 188.8972797 & 176.1675893 & 167.1876435 & 160.5133131 \\
\hline
\end{tabular}

Similarly in $t_{C 1, j}, j=1,2, \ldots, 10$, it is shown that the proposed family of estimators, $t_{C 2, j}, j=1,2, \ldots, 10$, perform better than compared estimators, $t_{R 2}, t_{\text {reg } 2}$ and $t_{\exp 2}$, under the Case II for both populations.

These results show that the proposed families of estimators $t_{C 1, j}$ and $t_{C 2, j}, j=1,2, \ldots, 10$ can be applied for estimating the population mean in the agriculture field for both cases.

\section{Conclusions}

This article proposes a family of estimators using the exponential function on estimation of the population mean in the presence of non-response. Firstly, we obtain the theoretical inferences and comparisons for the estimators under the Case I and Case II and then we found that the $t_{C 1, j}$ and $t_{C 2, j}, j=1,2, \ldots, 10$ estimators are more efficient than others in literature under the obtained conditions for both cases. In empirical studies, we use real data sets with the aim of showing the appropriateness of estimators in agriculture. According to the obtained results, the proposed family of estimators can appropriately be used in the agriculture on the estimation of the population mean. 


\section{Acknowledgements}

This publication is a part of $\mathrm{PhD}$ thesis of the first author.

\section{References}

Bahl S, Tuteja R K (1991). Ratio and product type exponential estimators. Journal of Information and Optimization Sciences 12(1): 159-164. https://doi.org/10.1080/02522667.1991.10699058

Cochran W G (1977). Sampling Techniques, John Wiley and Sons, New York.

Dansawad N (2019). A Class of Exponential Estimator to Estimate the Population Mean in the Presence of Non-Response. Naresuan University Journal: Science and Technology (NUJST) 27(4): 20-26. https://doi.org/10.14456/nujst.2019.33

Hansen M H \& Hurwitz W N (1946). The problem of non-response in sample surveys. Journal of the American Statistical Association 41(236): 517-529. https://doi.org/10.1080/01621459.1946.10501894

Khare B B \& Sinha R R (2009). On class of estimators for population mean using multi-auxiliary characters in the presence of nonresponse. Statistics in Transition 10(1): 3-14

Khare B B \& Srivastava S (1993). Estimation of population mean using auxiliary character in presence of non-response. National Academy Science Letters, India 16: 111-114

Kumar S (2013). Improved exponential estimator for estimating the population mean in the presence of non-response. Communications for Statistical Applications and Methods 20(5): 357-366. https://doi.org/10.5351/csam.2013.20.5.357

Kumar S \& Bhougal S (2011). Estimation of the population mean in presence of non-response. Communications for Statistical Applications and Methods 18(4): 537-548. https://doi.org/10.5351/ckss.2011.18.4.537

Kumar K \& Kumar M (2017). Improved exponential ratio and product type estimators for population mean in the presence of nonresponse. Bulletin of Mathematics and Statistics Research 5(2): 68-76

Pal S K \& Singh H P (2016). Finite population mean estimation through a two-parameter ratio estimator using auxiliary information in presence of non-response. Journal of Applied Mathematics, Statistics and Informatics 12(2): 5-39. https://doi.org/10.1515/jamsi-2016-0006

Pal S K \& Singh H P (2017). A class of ratio-cum-ratio-type exponential estimators for population mean with sub sampling the nonrespondents. Jordan Journal of Mathematics and Statistics 10(1): 73-94

Pal S K \& Singh H P (2018). Estimation of finite population mean using auxiliary information in presence of non-response. Communications in Statistics-Simulation and Computation 47(1): 143-165. https://doi.org/10.1080/03610918.2017.1280161

Rao P S R S (1986). Ratio estimation with sub sampling the non-respondents. Survey Methodology 12: 217-230

Riaz S, Nazeer A, Abbasi J \& Qamar S (2020). On the generalized class of estimators for estimation of finite population mean in the presence of non-response problem. Journal of Prime Research in Mathematics 16(1): 52-63

Solanki R S, Singh H P \& Rathour A (2012). An alternative estimator for estimating the finite population mean using auxiliary information in sample surveys. International Scholarly Research Notices 1-14. https://doi.org/10.5402/2012/657682

Singh R, Kumar M, Chaudhary M K \& Smarandache F (2009). Estimation of mean in presence of non-response using exponential estimator. Unpublished manuscript. arXiv preprint arXiv:0906.2462.

Singh R, Mishra P, Auduudu A \& Khare S (2020). Exponential type estimator for estimating finite population mean. International Journal of Computational and Theoretical Statistics 7(1): 37-41

Singh G N \& Usman M (2019a). Ratio-to-product exponential-type estimators under non-response. Jordan Journal of Mathematics and Statistics 12(4): 593-616

Singh G N \& Usman M (2019b). Efficient combination of various estimators in the presence of non-response. Communications in StatisticsSimulation and Computation 1-35. https://doi.org/10.1080/03610918.2019.1614618

Unal C \& Kadilar C (2020). Exponential type estimator for the population mean in the presence of non-response. Journal of Statistics and Management Systems 23(3): 603-615. https://doi.org/10.1080/09720510.2019.1668158

Unal C \& Kadilar C (2021). Improved family of estimators using exponential function for the population mean in the presence of nonresponse. Communications in Statistics - Theory and Methods 50(1): 237-248. https://doi.org/10.1080/03610926.2019.1634818

Yadav S K, Subramani J, Misra S, Singh L \& Mishra S S (2016). Improved estimation of population mean in presence of non-response using exponential estimator. International Journal of Agricultural and Statistical Sciences 12(1): 271-276

Olufadi Y \& Kumar S (2014). Ratio-cum-product estimator using exponential estimator in the presence of non-response. Journal of Advanced Computing 3(1): 1-11. https://doi.org/10.7726/jac.2014.1001

(C) 2022 by the author(s). Published by Ankara University, Faculty of Agriculture, Ankara, Turkey. This is an Open Access article distributed under the terms and conditions of the Creative Commons Attribution (CC BY) license (http://creativecommons.org/licenses/by/4.0/), which permits unrestricted use, distribution, and reproduction in any medium, provided the original work is properly cited. 